Carnets de géographes

GÉOGRAPHES

$10 \mid 2017$

Pour une réflexion collective sur l'enseignement de la géographie à l'Université

\title{
L'effet levier dans la reconversion des sites militaires dans le cadre du renouvellement urbain en France
}

Stéphanie Lotz

\section{OpenEdition}

Journals

Édition électronique

URL : http://journals.openedition.org/cdg/1201

DOI : 10.4000/cdg. 1201

ISSN : $2107-7266$

\section{Éditeur}

UMR 245 - CESSMA

Référence électronique

Stéphanie Lotz, «L'effet levier dans la reconversion des sites militaires dans le cadre du

renouvellement urbain en France », Carnets de géographes [En ligne], 10 | 2017, mis en ligne le 01

octobre 2017, consulté le 24 septembre 2020. URL : http://journals.openedition.org/cdg/1201 ; DOI https://doi.org/10.4000/cdg.1201

Ce document a été généré automatiquement le 24 septembre 2020

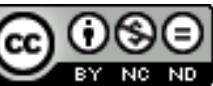

La revue Carnets de géographes est mise à disposition selon les termes de la Licence Creative Commons Attribution - Pas d'Utilisation Commerciale - Pas de Modification 4.0 International. 


\title{
L'effet levier dans la reconversion des sites militaires dans le cadre du renouvellement urbain en France
}

\author{
Stéphanie Lotz
}

1 En France, dans un contexte de réforme générale des politiques publiques et d'adaptation de l'outil de Défense aux nouvelles contraintes géostratégiques, les friches militaires sont de plus en plus nombreuses et la cessation de leurs activités représente souvent un traumatisme économique pour le territoire concerné. Il s'agit de sites qui, lorsqu'ils étaient en activité, appartenaient au ministère de la défense. Leur nature peut revêtir des aspects très divers, casernes urbaines, fortifications héritées de Vauban, champs de tir et camps d'entraînement en rase campagne, bases aériennes ou même usine de production d'armement. Dans tous les cas, leurs activités, administratives ou d'entraînement des militaires, faisaient généralement l'objet de beaucoup de discrétion et se développaient de façon autonome.

2 La reconversion de ces friches coïncide souvent avec un changement de statut, le ministère de la défense cédant son bien dans des conditions diverses pour favoriser son changement d'usage.

3 La reconversion de ces sites se heurte alors fréquemment à des stéréotypes concernant le site en lui-même, du fait de l'ignorance des activités qui s'y étaient déroulées, y compris pour les friches militaires urbaines. Au-delà de cette première barrière, le fait même de considérer la friche militaire comme un site extraordinaire au sens premier du terme semble constituer un second frein à la reconversion. Alors que ces sites sont mis sur le marché foncier de façon accrue depuis les années 2000, ils sont pourtant souvent le seul foncier disponible dans le cadre du renouvellement urbain pour une ville qui se veut compacte.

4 Ce travail de thèse a permis d'interroger la banalisation de la friche militaire urbaine en élaborant une méthode statistique considérant l'ensemble des friches militaires mises sur le marché foncier par le ministère de la défense depuis les années 1980. Il a alors été possible de démontrer que la friche militaire urbaine, à l'issue de sa cessation 
d'activité et dès lors qu'elle s'inscrit dans une logique intramuros, ou sur les franges de la ville, peut être considérée comme une friche quelconque, sa nature, ses modalités de cession et enfin sa réintégration dans la trame urbaine ne présentant pas de caractéristiques trop exclusives.

Figure 1 : la banalisation de la friche militaire passé le temps de veille

\section{La banalisation de la friche militaire passé le temps de veille}

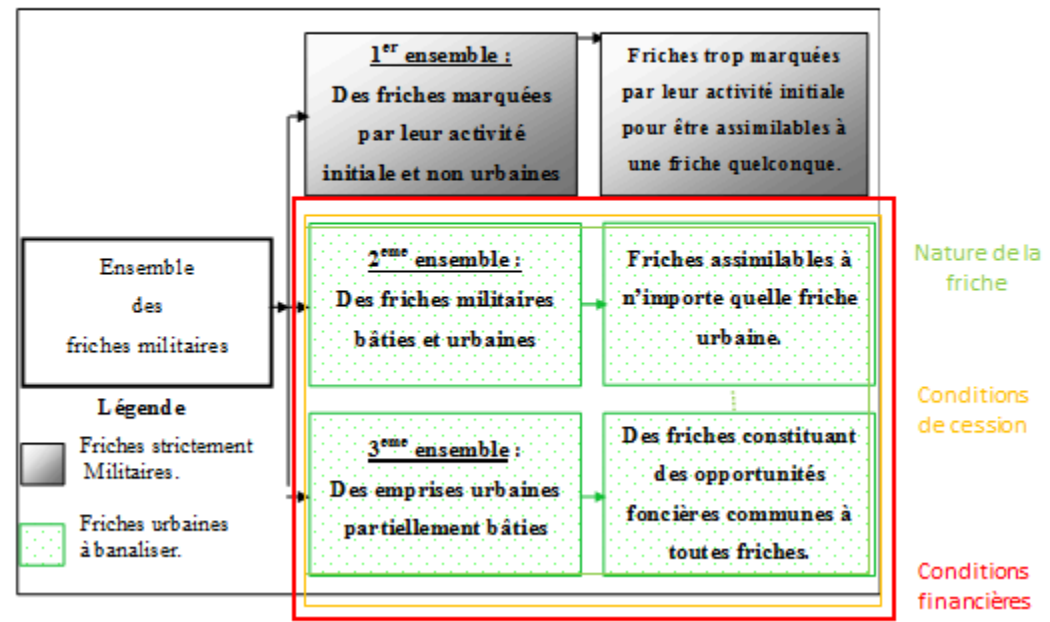

Réalisation auteure

Lorsque la fermeture de sites militaires est annoncée par voie médiatique, les élus et les acteurs économiques locaux argumentent immédiatement sur les pertes d'emplois, directs et indirects, de compétitivité et d'attractivité pour le territoire. Dans un tel contexte, la reconversion du site en cessation d'activité peut a priori paraître difficile et une vision pessimiste peut parfois s'installer quant à l'avenir du territoire concerné.

6 Le deuxième objectif de ce travail de recherche a donc été de vérifier dans quelle mesure les projets de reconversion des sites militaires pouvaient être des leviers puissants d'attractivité lorsqu'ils répondent aux besoins du territoire et à l'enjeu urbanistique de renouvellement urbain. Pour ce faire, la notion d'effet levier, technique destinée à multiplier les profits et les pertes sur la base d'un investissement initial, a été choisie. Dans le cadre d'une méthode inductive basée sur la sélection de cinq terrains d'étude (Noyon, Joigny, Langres, Grenoble et Mulhouse), il a été déterminé que la requalification d'une friche militaire urbaine peut avoir un effet levier qui se décline en trois aspects principaux: effets sur l'image de la ville, résultats économiques et sociaux favorables et enfin rétablissement d'une cohérence dans l'urbanité de la ville. Une ville peut donc tirer profit de la requalification d'un site militaire lorsqu'elle est réalisée dans le cadre d'un projet urbain de reconversion de la friche qui répond aux besoins du territoire précédemment identifiés. La reconversion pourrait alors positionner un territoire en permettant aux espaces concernés d'acquérir une nouvelle identité.

7 Afin de pouvoir parvenir à cette conclusion, il a fallu déterminer quelles devaient être les conditions favorables pour qu'une friche militaire urbaine reconvertie puisse effectivement avoir un effet levier pour la ville ou le territoire concernée. Dans un premier temps, les évolutions urbaines récentes et les études de terrain ont confirmé 
que la reconversion d'une friche n'est pas acquise a priori pour un terrain militaire mais qu'elle doit s'envisager dans le cadre d'un projet d'ensemble pour la Ville. Il est ainsi apparu que la notion de projet urbain est une première condition sine qua non pour la réussite de la reconversion d'un site militaire. Les études de terrain ont également montré que, si le projet urbain est un processus qui permet aux friches militaires d'être reconverties dans la logique d'un projet cohérent, il doit intégrer d'autres facteurs pour être à l'origine du gain d'attractivité. Ces éléments complémentaires se déclinent de deux façons : d'une part, avec une déclinaison plus fine de certaines composantes du projet urbain et, d'autre part, en intégrant des points spécifiques qui peuvent aussi s'assimiler à des éléments de contexte.

Les recherches liées à l'effet levier de la reconversion d'un site militaire nécessitaient d'envisager le temps de l'après-friche, une fois que la reconversion est réalisée, mais surtout de dépasser cette période et de se positionner dans le temps des résultats objet de peu de travaux. Dans le cadre de ce travail de recherche, il a donc été choisi d'en faire un quatrième temps de la friche nommé le temps de la friche reconvertie. Les études de terrain ont ensuite permis de découper ce nouveau temps de la friche en trois périodes (cf. figure 2) qui pourraient s'appliquer quel que soit le type de friche reconvertie considérée avec des limites temporelles qui mériteraient d'être affinées au cas par cas.

Figure 2 : les trois périodes du temps de la friche reconvertie

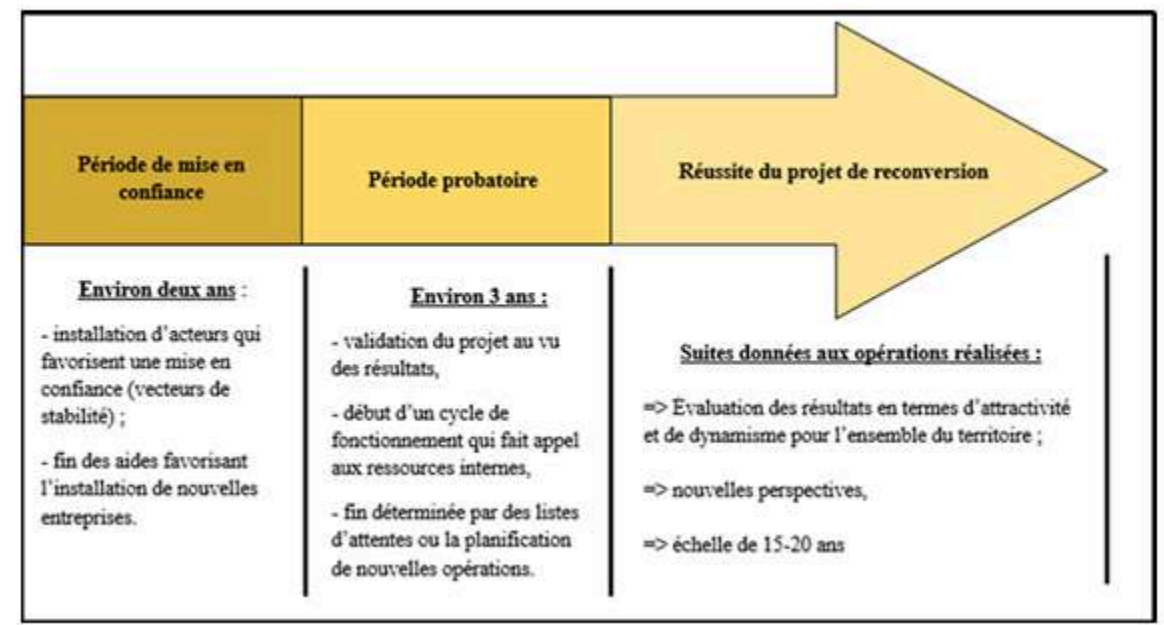

Réalisation auteure

\section{Discipline}

aménagement de l'espace et urbanisme

\section{Directrice de thèse}

Sonia Guelton

\section{Université}

Lab'Urba, l'École Doctorale Ville, Transports et Territoires, École d'urbanisme de paris, Paris XII 
Membres du jury de thèse soutenue publiquement le vendredi 13 janvier 2017

Mme Aurélie Delage, Maître de conférences, Université de Perpignan via Domitia, examinateur,

M. Jean-Claude Driant, Professeur, École d'urbanisme de Paris Université Paris-Est, examinateur

Mme Édith Fagnoni, Professeure, Université Paris-Sorbonne, rapporteur

Mme Sonia Guelton, Professeure, École d'urbanisme de Paris, Université Paris-Est, directrice de thèse

M. Didier Paris, Professeur, Université Lille 1, rapporteur

Situation professionnelle à l'issue de la thèse

chercheur associé au Lab'urba, École d'urbanisme de Paris, Paris XII

\section{Courriel}

stephanie.lotz[at]u-pec.fr

INDEX

Thèmes : Carnets de soutenances

Mots-clés : friche, ville, France

Keywords : Wasteland, Urban Space, France 